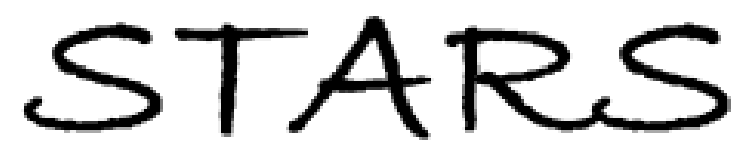

University of Central Florida

STARS

$1-1-2006$

\title{
SiC-based optical interferometry at high pressures and temperatures for pressure and chemical sensing
}

Surendramohan Dakshinamurthy

University of Central Florida

Nathaniel R. Quick

Aravinda Kar

University of Central Florida

Find similar works at: https://stars.library.ucf.edu/facultybib2000

University of Central Florida Libraries http://library.ucf.edu

This Article is brought to you for free and open access by the Faculty Bibliography at STARS. It has been accepted for inclusion in Faculty Bibliography 2000s by an authorized administrator of STARS. For more information, please contactSTARS@ucf.edu.

\section{Recommended Citation}

Dakshinamurthy, Surendramohan; Quick, Nathaniel R.; and Kar, Aravinda, "SiC-based optical interferometry at high pressures and temperatures for pressure and chemical sensing" (2006). Faculty Bibliography 2000s. 4684.

https://stars.library.ucf.edu/facultybib2000/4684

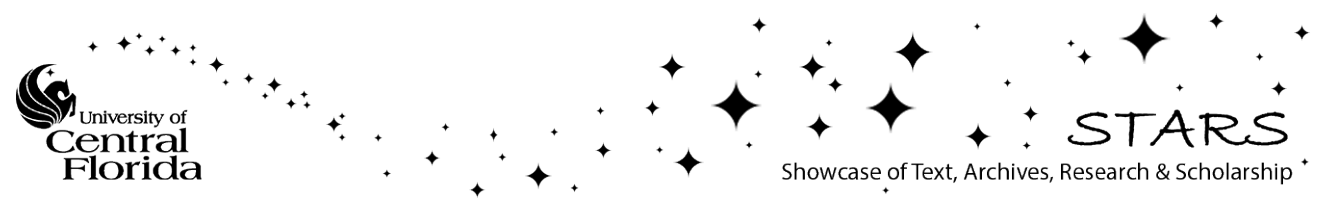




\section{Sic-based optical interferometry at high pressures and temperatures for pressure and chemical sensing}

Cite as: J. Appl. Phys. 99, 094902 (2006); https://doi.org/10.1063/1.2191478

Submitted: 29 November 2005 . Accepted: 19 February 2006 . Published Online: 11 May 2006

Surendramohan Dakshinamurthy, Nathaniel R. Quick, and Aravinda Kar

\section{ARTICLES YOU MAY BE INTERESTED IN}

Decoupling of silicon carbide optical sensor response for temperature and pressure measurements

Journal of Applied Physics 102, 073111 (2007); https://doi.org/10.1063/1.2786889

Piezoelectric properties and elastic constants of $4 \mathrm{H}$ and $6 \mathrm{H} \mathrm{SiC}$ at temperatures 4-320 K Journal of Applied Physics 66, 3922 (1989); https://doi.org/10.1063/1.344477

The elastic constants of silicon carbide: A Brillouin-scattering study of $4 \mathrm{H}$ and $6 \mathrm{H}$ SiC single crystals

Journal of Applied Physics 82, 3152 (1997); https://doi.org/10.1063/1.366100
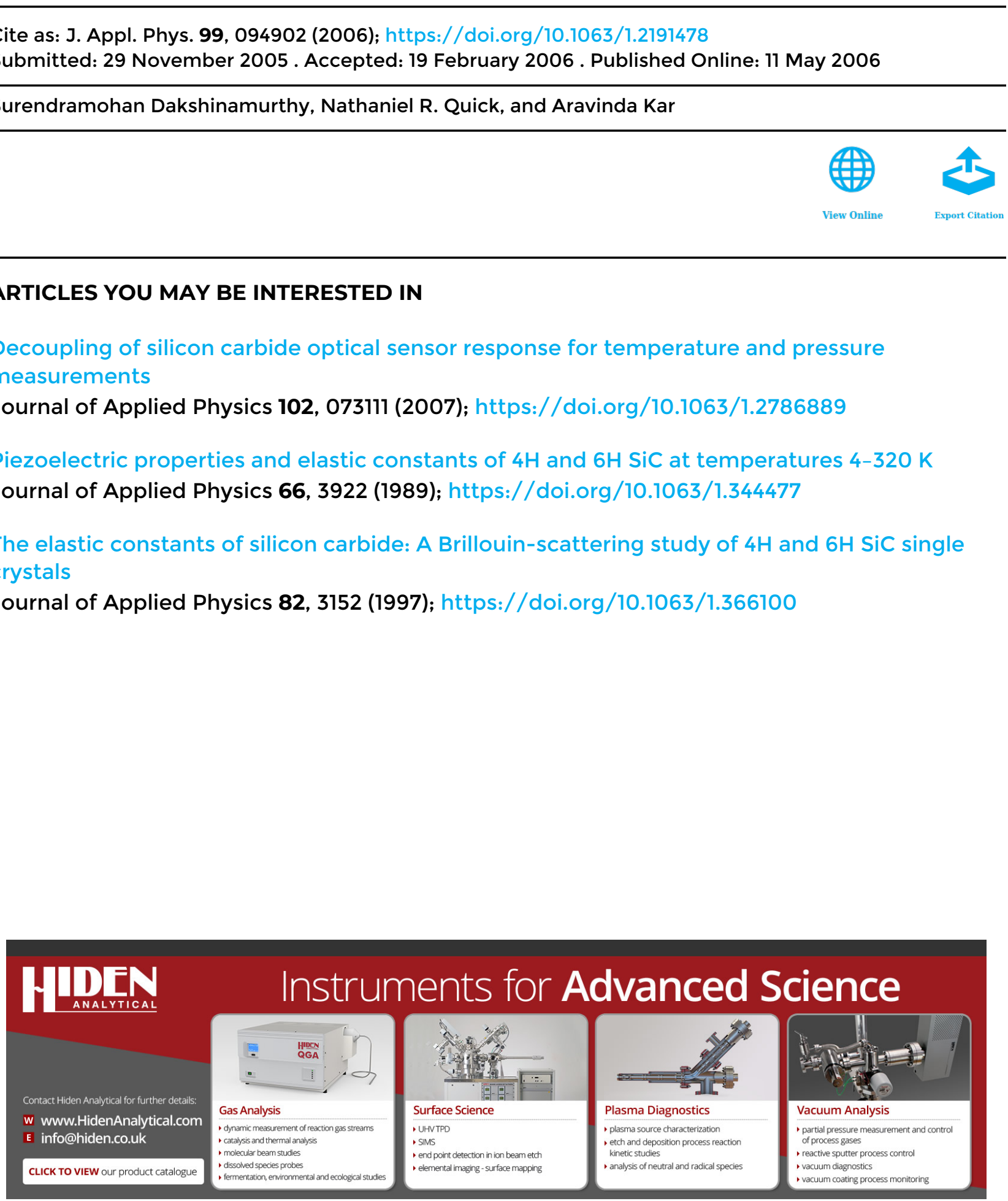


\title{
SiC-based optical interferometry at high pressures and temperatures for pressure and chemical sensing
}

\author{
Surendramohan Dakshinamurthy \\ Laser-Aided Manufacturing, Materials and Micro-Processing Laboratory (LAMMMP), College of Optics \\ and Photonics/CREOL, Department of Electrical Engineering and Computer Science, University \\ of Central Florida, Orlando, Florida 32816-2700
}

Nathaniel R. Quick

AppliCote Associates, LLC, 1445 Dolgner Drive, Suite 23, Sanford, Florida 32771

\author{
Aravinda Kar ${ }^{\mathrm{a}}$ \\ Laser-Aided Manufacturing, Materials and Micro-Processing Laboratory (LAMMMP), College of Optics \\ and Photonics/CREOL, Mechanical, Materials, and Aerospace Engineering Department, University \\ of Central Florida, Orlando, Florida 32816-2700
}

(Received 29 November 2005; accepted 19 February 2006; published online 11 May 2006)

\begin{abstract}
Crystalline silicon carbide is a chemically inert wide band gap semiconductor with good mechanical strength and oxidation-resistant properties at elevated temperatures, which make it a good sensor material for harsh environments such as combustion chambers and turbine systems. For such cases, optical sensors are generally superior to electrical sensors in many aspects such as responsivity, detectivity, and sensitivity. This paper presents a wireless technique for pressure and chemical sensing based on the pressure-and temperature-dependent refractive indices of silicon carbide. A helium-neon laser with a wavelength of $632.8 \mathrm{~nm}$ was used as a probe laser to obtain the complementary Airy pattern of the laser power reflected off a silicon carbide wafer segment at high temperatures (up to $300{ }^{\circ} \mathrm{C}$ ) and pressures (up to $400 \mathrm{psi}$ ). The interference patterns revealed unique characteristics for nitrogen and argon test gases. This pattern is different at the same pressure and temperature for the two gases, indicating the chemical sensing selectivity capability of silicon carbide. Also the pattern changes with pressures for the same gas, indicating the pressure sensing capability. The refractive index of silicon carbide has been obtained for different pressures and temperatures using the interference pattern. A three-layer model has been employed to determine the refractive indices of the gases using the reflected power data. (C) 2006 American Institute of Physics.

[DOI: $10.1063 / 1.2191478]$
\end{abstract}

\section{INTRODUCTION}

Sensors are used for pressure and temperature measurements as well as for detecting gases. Various types of sensors are available, each having operational capability within a certain limit. The combustion chambers and turbine systems in most of the power plants operate with an average efficiency of about 33\%. The efficiency can be increased by operating the power plants at very high pressures and high temperatures and by implementing advanced feedback process control electronics such as the sensors. Gas sensors and their sensing mechanism for such applications are under active investigation. ${ }^{1-6}$

Most of these sensors are based on silicon, thus rendering their operational limits to below $250{ }^{\circ} \mathrm{C}^{7}$ Some of the key requirements of the sensor are its ability to withstand harsh environment during sensing operation and its response time. The lambda sensor for measuring the gas concentration in automobile engines is not operated during the startup of the engine, because contact with engine fluids could damage the hot sensor. ${ }^{8}$ About $98 \%$ of the pollutants are released during this startup period resulting from the absence of a sensing capability. Improved sensing capability can be real-

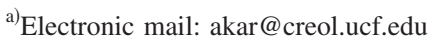

ized by replacing current silicon sensors with wide band gap semiconductor-based sensors that are capable of withstanding harsh environmental conditions.

$\mathrm{SiC}$ is considered to be a high temperature and high power electronic material due to its superior thermal, mechanical, and electrical properties. The band gap of $6 \mathrm{H}-\mathrm{SiC}$ (around $3 \mathrm{eV})^{9}$ is more than twice the band gap of silicon. The high sublimation temperature (about $2700{ }^{\circ} \mathrm{C}$ ), extremely low intrinsic carrier concentration, and wide band gap allow SiC-based sensors to operate at elevated temperatures, without a significant degradation of the electronic and other material properties. ${ }^{10}$ It is not possible to achieve such operational capability with conventional Si-based devices. $\mathrm{SiC}$ sensors can also endure corrosive media with minimal degradation due to their chemical inertness, thereby enabling its usage in a variety of sensing applications where Si-based components fail. The strong affinity of $\mathrm{SiC}$ to hydrocarbon makes it suitable for gas sensor applications. ${ }^{11}$

The semiconducting property of $\mathrm{SiC}$ has been exploited widely for sensor design. Field effect transistor (FET)-based devices are used in numerous applications such as combustion furnace, automobile industry, selective catalytic reduction (SCR) systems, and also as lambda sensors. ${ }^{12}$ The sensor response time, which is a critical parameter in feedback pro- 
cess control applications, has been tested for various configurations of catalytic metals used in FET-based sensors. The time constant of the sensors was found to be very small and heavily influenced by temperature. Prototypes of such sensors exhibited a response time less than $10 \mathrm{~ms}$ at $500{ }^{\circ} \mathrm{C}$ when changing from an oxidizing to a reducing atmosphere. ${ }^{13}$ For small power plants $(\sim 1 \mathrm{MW})$, Fourier transform infrared (FTIR) spectroscopy is an expensive technique; so sensors based on electrical response are viable alternatives. SiC-based metal-semiconductor field-effect transistor (MESFET) sensors, with proper choice of the type and structure of the catalytic gate, can be used for sensing applications. Metal, reactive insulator-based sensors have also been developed for hydrocarbon sensing with an operational temperature range of $300-600{ }^{\circ} \mathrm{C} .{ }^{14}$ The performance of sensors that are based on the electrical response is affected by the contact lead degradation and the chemical reactions between the metal and semiconductor. At high temperatures the electrical contact to the sensor tends to melt and silicides and carbides tend to form at the metal-SiC interface. Sensors that are based on optical response ${ }^{15-19}$ offer advantages in this regard. Optical techniques such as multibeam interference have been used for temperature measurement by interrogating a thin film of $\mathrm{SiC}(0.5-2 \mu \mathrm{m})$ on a single crystal sapphire, a Fabry-Pérot cavity. The temperature response of the sensor was evaluated up to $540{ }^{\circ} \mathrm{C}$ in the visible to infrared wavelength region. ${ }^{20}$ In this study the optical response of a silicon carbide wafer segment has been detected in the form of a complementary Airy pattern for two different gases for varying temperature and pressure. The SiC wafer segment is a Fabry-Pérot etalon. The principle of multibeam interferometry has been applied to analyze the patterns.

\section{EXPERIMENTAL PROCEDURE}

Single crystal silicon carbide $(6 H$-SiC polytype) wafer segments were used in this study. The thickness of the wafer was measured to be $420 \mu \mathrm{m}$. The wafer was cleaned chemically before conducting reflected power measurement experiments. A $1 \mathrm{~min}$ buffered oxide etching was carried out to etch any native oxide that might have formed on the surface of the wafer. The formation of oxide scale is similar to the case in silicon except that it is much slower in SiC. The etching process was carried out to ensure that the wafer was clean. The sample was rinsed with de-ionized water, cleaned with soap scrub to remove any dirt particles, followed by rinsing with trichloroethylene, acetone, methanol (TAM), and de-ionized water to remove organic contaminants from the surface. Excess de-ionized water was removed using neutral absorbent paper and air dried using a nitrogen gun.

The schematic of the experimental setup used for studying the temperature-dependent and pressure-dependent optical properties of silicon carbide for two different gases is shown in Fig. 1. It provides various features of a laser-based wireless gas sensing system. The sensor is a polished $\mathrm{SiC}$ wafer segment with polished surfaces to allow specular reflection of the He-Ne laser beam. It is placed on a shallow groove of diameter $2.54 \mathrm{~cm}$ in a stainless steel pedestal of diameter $2.9 \mathrm{~cm}$. The pedestal is placed at the center of a

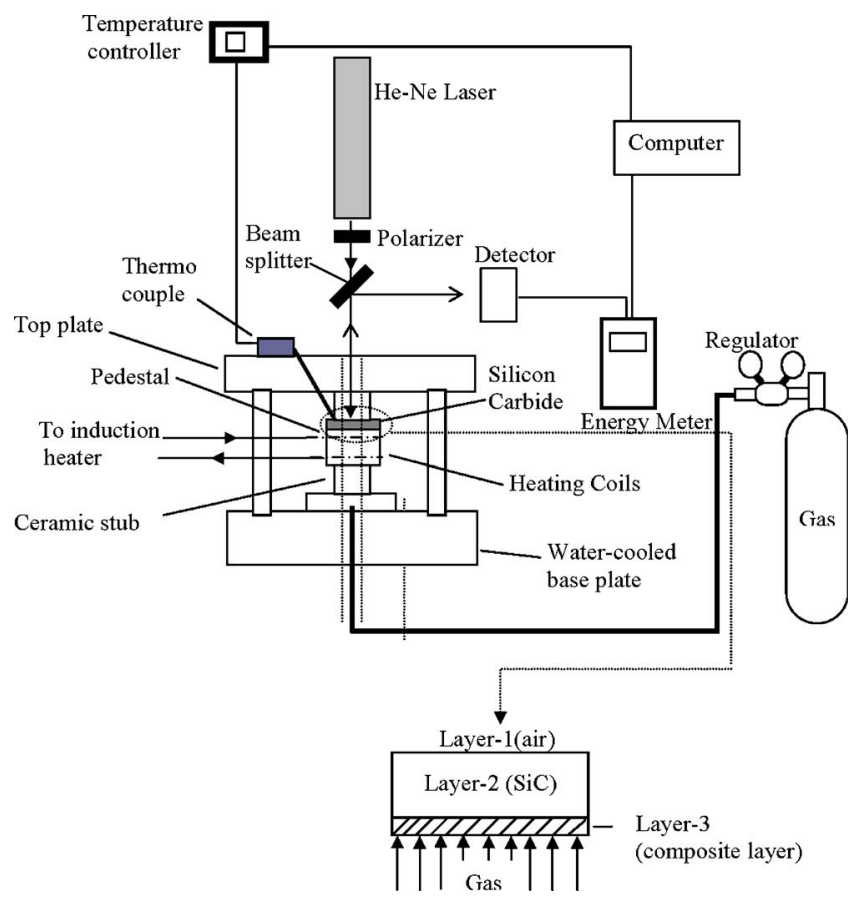

FIG. 1. Schematic layout of the experimental setup used for reflectivity measurement.

water-cooled copper coil connected to a water-cooled induction heater to heat the pedestal uniformly. The hot pedestal heats up the sensor. The pedestal contains a small hole of $7.9 \mathrm{~mm}$ diameter that creates a $50 \mathrm{~mm}^{2}$ area for the reflected power interrogation measurement. The volume of the hole beneath this sensor area represents the ambient whose property needs to be monitored with the sensor in practical applications. This volume can be filled with different gases through a gas feed mechanism and the pressure of the gas is controlled with a high pressure regulator. To hold the sensor in place against this gas pressure, a ceramic insulator was placed on top of the sensor and then a copper plate was mounted on the ceramic insulator. The copper plate was secured to three copper posts attached to a stainless steel base plate. The copper plate and the ceramic insulator have a hole at the center similar to the pedestal to interrogate the sensor.

A continuous wave $\mathrm{He}-\mathrm{Ne}$ laser with a wavelength of $632.8 \mathrm{~nm}$ was used as an interrogation probe beam to examine the reflective property of the $\mathrm{SiC}$ sensor as a function of temperature, gas, and gas pressure. The reflected power was measured with an optical power detector connected to a power meter. The temperature of the sensor was measured with a thermocouple connected to a temperature controller. Both the reflected power and temperature were collected in a time-synchronized manner through computerized data acquisition. The randomly polarized $\mathrm{He}-\mathrm{Ne}$ laser beam was converted to the transverse magnetic (TM) mode, i.e., a parallel polarization component created by passing the beam through a polarizer. For normal incidence of the He-Ne beam, the TM polarized beam is partially transmitted through a beam splitter placed at a $45^{\circ}$ angle with respect to the incident beam. The beam splitter was specifically designed to operate at $632.8 \mathrm{~nm}$ wavelength. A fraction of this beam is reflected 
and transmitted by the sensor. The reflected beam is transmitted through the beam splitter onto a detector that measures its power.

\section{RESULTS AND DISCUSSION}

The optical properties of crystalline solids are generally related to their response to any optical excitation at a given temperature and pressure. While the stainless steel pedestal in Fig. 1 enables heating the $\mathrm{SiC}$ wafer segment gas pressure can be applied to the bottom surface of the wafer segment interfacing a pressure cell. The effects of various gases and their pressures are studied by examining the reflective property of the wafer segment. The power of the He-Ne laser beam reflected by the silicon carbide sample exhibited oscillatory patterns (Figs. 2 and 3), which are due to the constructive and destructive interferences of the reflected light. A portion of the incident beam is transmitted through the silicon carbide wafer segment. This beam undergoes multiple reflections between the top and bottom surfaces of the wafer segment, emerges as phase-shifted light through the top surface, and interferes to produce the observed interference pattern. In Figs. 2 and 3, the maxima represent the constructive interference effect and the minima are the destructive interference. The maxima correspond to the even multiples of the phase angle $(\phi)$ and the minima correspond to the odd multiples of $\phi$. For normal incidence of the laser beam, the phase angle is given by

$$
\varphi=2 \pi \frac{n(\lambda, T, P) d(T, P)}{\lambda},
$$

where $n(\lambda, T, P)$ is the refractive index of the silicon carbide wafer segment, which is influenced by the wavelength $(\lambda)$ due to the dispersion phenomenon, temperature $(T)$ due to the thermo-optic effect, and pressure $(P)$ due to the stressoptics effect. $d(T, P)$ is the sample thickness which is affected by the temperature and pressure, and $\lambda$ is the wavelength of the incident light in vacuum.

Figures 2 and 3 represent the reflected power of the $\mathrm{SiC}$ wafer segment when its bottom surface is exposed to nitrogen and argon, respectively, at varying pressures. The change in temperature at a constant pressure causes the reflected power to oscillate between certain maximum and minimum values. These oscillations reveal an interesting pattern. Unlike the cases of atmospheric pressure [Figs. 2(a) and 3(a)], the oscillations tend to diverge progressively with temperature at higher pressures. The divergence patterns of the oscillations are unique or to the type of gases, nitrogen and argon in this case, signifying that these patterns can be attributed to the characteristic identity of the individual gases in chemical sensing applications. The sensor system is selective to the gas species. Another aspect of these characteristic oscillations is that the divergence pattern fans out with increasing pressure, thus enabling pressure sensing capability of the sensor.

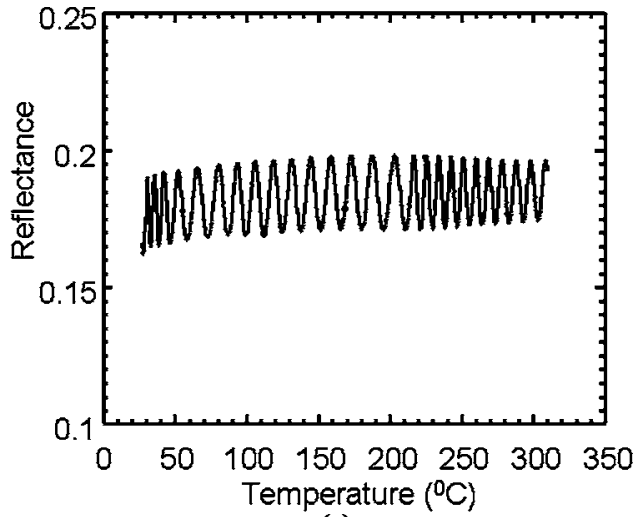

(a)

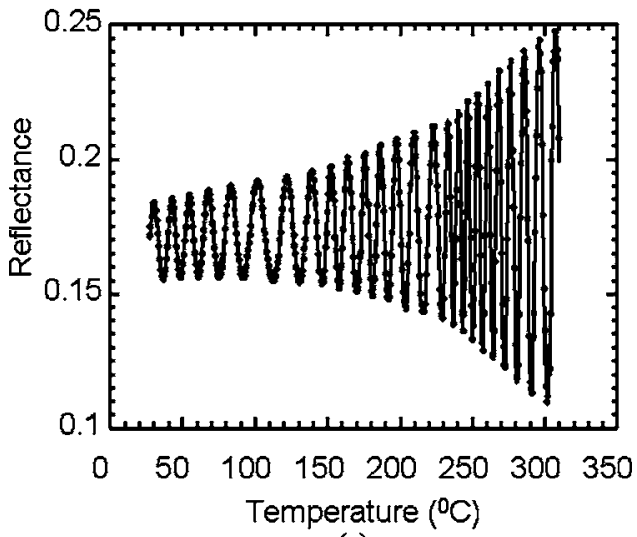

(c)

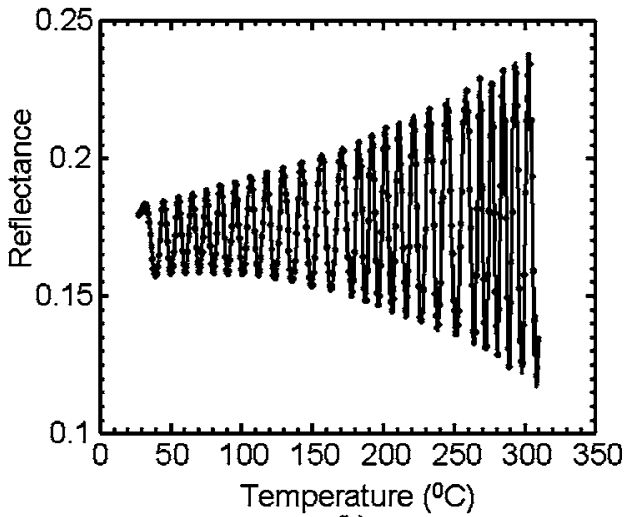

(b)

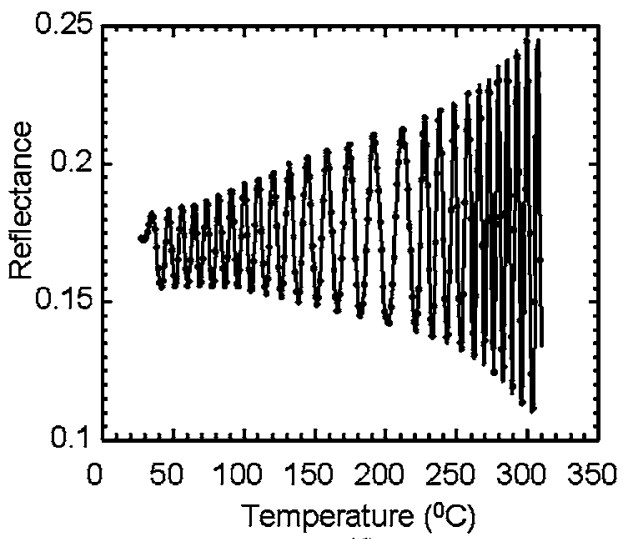

(d)
FIG. 2. Reflectance of $6 \mathrm{H}$-SiC upon exposure to nitrogen gas at pressures of (a) 14.7, (b) 100, (c) 200, and (d) $400 \mathrm{psi}$, as a function of temperature from 27 up to $300{ }^{\circ} \mathrm{C}$ for normal incidence of the laser beam. 


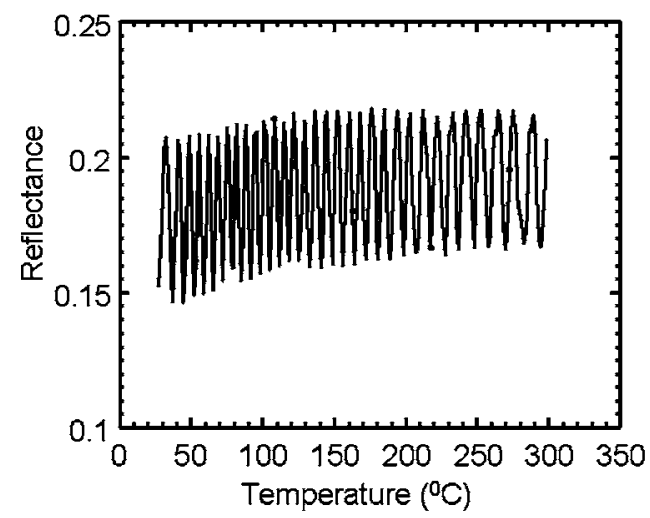

(a)

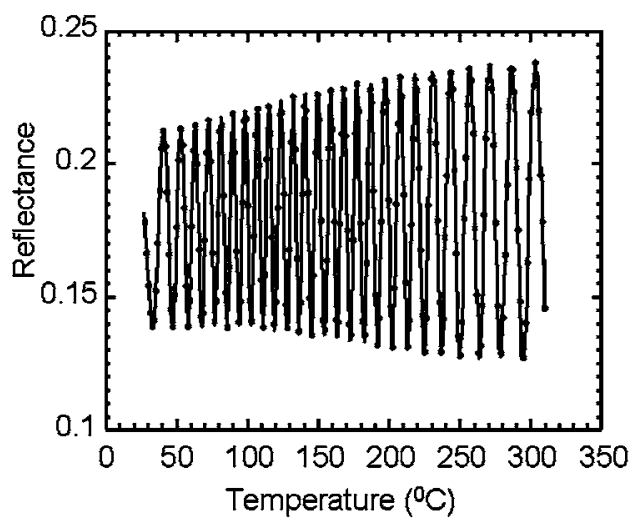

(c)

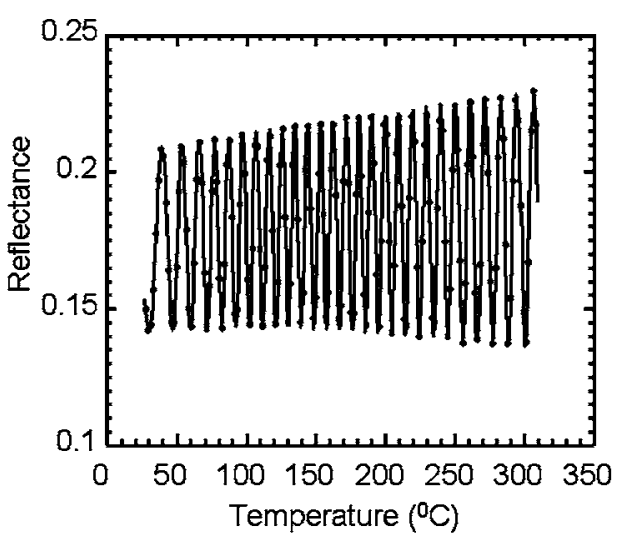

(b)

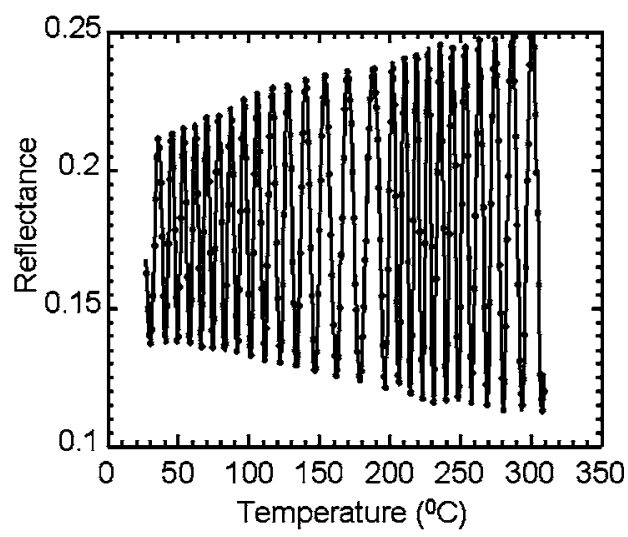

(d)
FIG. 3. Reflected power of silicon carbide upon exposure to argon gas at pressures of (a) 14.7, (b) 100, (c) 200, and (d) $400 \mathrm{psi}$, as a function of temperature from 27 up to $300{ }^{\circ} \mathrm{C}$ for normal incidence of the laser beam.

\section{A. Refractive index of $6 \mathrm{H}-\mathrm{SiC}$ at high pressures and temperatures}

The temperature dependent refractive index ${ }^{21}$ of a sample can be obtained from the above-mentioned interference pattern using the following expression:

$$
n_{m+1}=\frac{1}{1+\alpha_{\text {mid }} \Delta T / 2}\left[\frac{\lambda_{0}}{2 d\left(T_{0}, P\right)}+\left(1-\frac{\alpha_{\text {mid }} \Delta T}{2}\right) n_{m}\right] \text {, }
$$

where $n_{m+1}$ and $n_{m}$ are the refractive indices of silicon carbide at locations $l_{m+1}$ and $l_{m}$, i.e., at temperatures $T_{m+1}$ and $T_{m}$, respectively (Fig. 4), and at the pressure $P$ that was applied to the wafer segment using nitrogen or argon. In other words, $n_{m}$ can be written as $n_{m}=n\left(T_{m}, P\right)$ for a fixed wavelength. $\alpha_{\text {mid }}$ is the thermal expansion coefficient of silicon carbide at the temperature corresponding to the midpoint $\left(l_{\text {mid }}\right)$ of the straight line $l_{m} l_{m+1}$ in Fig. 4. Its value for silicon carbide is given by $^{22}$

$$
\alpha=3.19 \times 10^{-6}+3.60 \times 10^{-9} \times T-1.68 \times 10^{-12} \times T^{2},
$$

so $\alpha_{\text {mid }}=\alpha\left[\left(T_{m}+T_{m+1}\right) / 2\right]$ and $\Delta T=T_{m+1}-T_{m}$. Knowing the refractive index $\left(n_{0}\right)$ of $\mathrm{SiC}$, which is calculated at room temperature $\left(T_{0}\right)$ using Fresnel's formula $R=(n-1)^{2} /$ $(n+1)^{2}$, the refractive indices can be obtained at higher temperatures from Eq. (2). While the effect of temperature on the thickness of silicon carbide is considered using the thermal expansion coefficient, the effect of pressure on the thick- ness is taken into account using the expression $d\left(T_{0}, P\right)$ $=d_{0}(1-\varepsilon)$, where $d\left(T_{0}, P\right)$ is the wafer segment thickness at room temperature $\left(T_{0}\right)$ and pressure $(P)$, whose values are approximately 412,405 , and $390 \mu \mathrm{m}$ at the gas pressures of 100,200 , and 400 psi, respectively and $d_{0}=420 \mu \mathrm{m}$ which is the original thickness of the wafer segment.

The strain $(\varepsilon)$ is given by ${ }^{23} \varepsilon=\sigma / E$, where $\sigma$ is the applied stress and $E$ is the elastic modulus. The value of elastic modulus has been reported as $392-694 \mathrm{GPa}$ for $6 \mathrm{H}$-SiC. ${ }^{24}$ In this study an average value of $543 \mathrm{GPa}$ was used for $E$

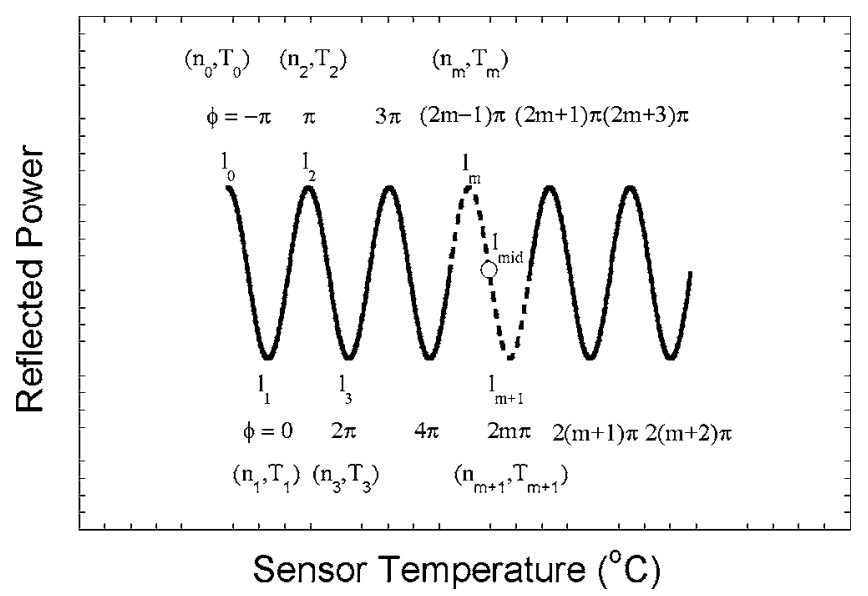

FIG. 4. A typical oscillatory reflected power profile (complementary Airy pattern) showing the phase angles $(\phi)$ between the adjacent branches of the oscillations. Such patterns are formed due to the interference of a multiply reflected $\mathrm{He}-\mathrm{Ne}$ laser beam. 


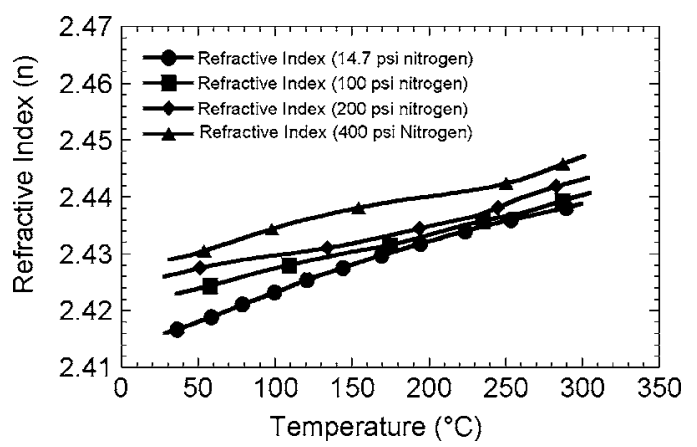

FIG. 5. Refractive index of silicon carbide when exposed to nitrogen at different pressures $(14.7,100,200$, and $400 \mathrm{psi})$ and temperatures (27 up to $\left.300^{\circ} \mathrm{C}\right)$.

and the stresses were taken to be 14.7, 100, 200, and 400 psi. It can be observed from Figs. 5 and 6 that the refractive index of silicon carbide increases with applied pressure. The interface of the silicon carbide wafer and gas (nitrogen or argon) experiences compressive stresses under high gas pressures and this produces a comparatively denser layer of silicon carbide near its bottom surface. The refractive index of this compressed layer is higher than the refractive index of the overlying uncompressed layer. This is evident in Figs. 5 and 6 showing an increase in the refractive index with pressure.

\section{B. Three-layer model for the effect of gas pressure on the refractive index of $6 \mathrm{H}$-SiC}

As mentioned above, the type of the gas and the pressure of the gas affect the oscillatory pattern of the reflected light.

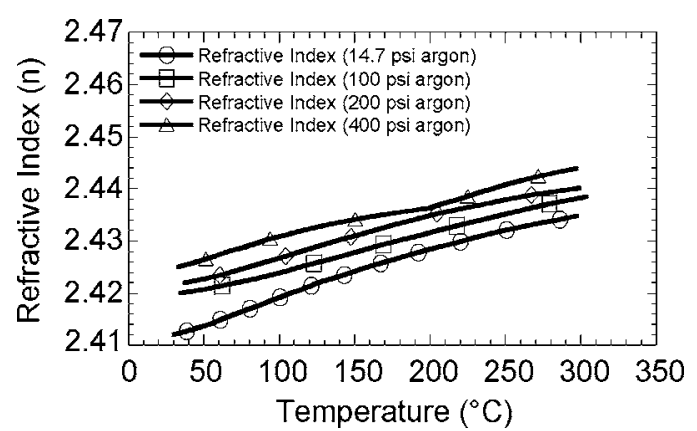

FIG. 6. Refractive index of silicon carbide when exposed to argon at different pressures $(14.7,100,200$, and $400 \mathrm{psi})$ and temperatures $\left(27-300{ }^{\circ} \mathrm{C}\right)$.

To analyze this effect, a three-layer model is considered (inset in Fig. 1) in which layer 1 is the ambient air on top of the $\mathrm{SiC}$ wafer, layer 2 is the $\mathrm{SiC}$ wafer itself with refractive index as determined above, and layer 3 is a composite layer consisting of a few compressed atomic planes of the $\mathrm{SiC}$ crystal and a high pressure sheet (boundary layer) of the gas around the bottom surface of the wafer. The reflectance, $R$, of such a three-layer structure can be expressed as ${ }^{25}$

$$
R=\frac{r_{12}^{2}+r_{23}^{2}+2 r_{12} r_{23} \cos \phi}{1+r_{12}^{2} r_{23}^{2}+2 r_{12} r_{23} \cos \phi}
$$

for a given laser beam, where $r_{12}$ and $r_{23}$ are the Fresnel reflection coefficients of layer 1-layer 2 and layer 2-layer 3 interfaces, respectively. Equation (4) can be solved for $r_{23}$, which is given by

$$
r_{23}=\frac{-\left[(1-R) r_{12} \cos \phi\right] \pm \sqrt{\left[(1-R) r_{12} \cos \phi\right]^{2}-\left(1-R r_{12}^{2}\right)\left(r_{12}^{2}-R\right)}}{\left(1-R r_{12}^{2}\right)}
$$

and then the refractive index, $n_{3}$, of the composite layer is obtained from the Fresnel relation expressing the refractive index in terms of the Fresnel reflection coefficient ${ }^{25}$

$$
n_{3}=n \frac{1+r_{23}}{1-r_{23}}
$$

Equation (5) yields two values of $r_{23}$, which in turn provide two values of the refractive index $n_{3}$. In this study, one of the two values was found to be very close to the refractive index of $\mathrm{SiC}$ (e.g., 2.39) and the other value was very high (e.g., 17.44). So the actual refractive index $n_{3}$ was selected to be the one that was within close proximity of the refractive index of $\mathrm{SiC}$.

To calculate $r_{23}$ using Eq. (6), the reflectance data (Figs. 2 and 3) were used and $r_{12}$ was determined using Fresnel's relation $r_{12}=\left(n_{2}-1\right) /\left(n_{2}+1\right)$. The value of $\phi$ at any point on the complementary Airy pattern was obtained through linear interpolation along each arm of the pattern (e.g., straight line $l_{m} l_{m+1}$ in Fig. 4) and by noting that the maximum and mini- mum points in the pattern correspond to even and odd multiples of $\pi$, respectively. The refractive index of the composite layer $\left(n_{3}\right)$ was obtained using Eq. (6) and its average values are shown in Figs. 7 and 8. As mentioned before, the

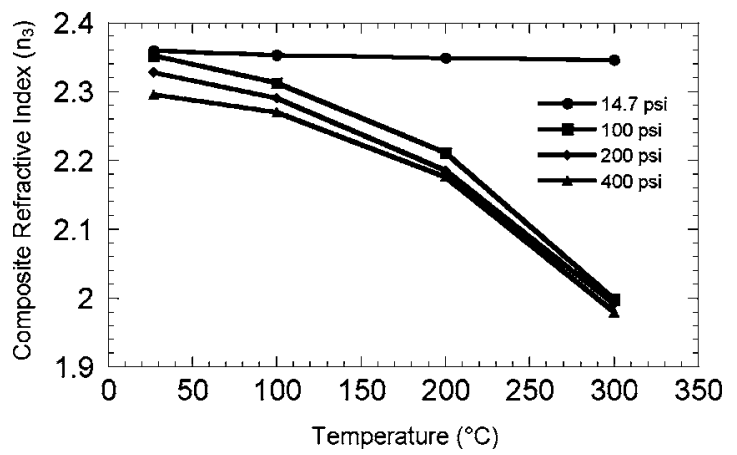

FIG. 7. Refractive index of the composite layer due to nitrogen at different pressures $(14.7,100,200$, and $400 \mathrm{psi})$ and temperatures from 27 up to $300{ }^{\circ} \mathrm{C}$. 


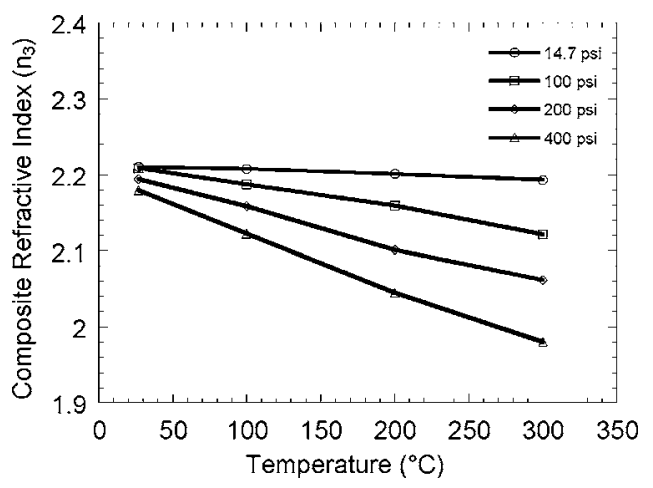

FIG. 8. Refractive index of the composite layer due to argon at different pressures $(14.7,100,200$, and $400 \mathrm{psi})$ and temperatures from 27 up to $300{ }^{\circ} \mathrm{C}$.

composite layer is formed by the SiC-gas interface (inset in Fig. 1) and therefore the optical properties of this region are expected to be different from that of the gas. Also the refractive index of this composite region may be governed by the complex interactions of the atoms of $\mathrm{SiC}$ and gas at high temperatures and pressures.

Figures 7 and 8 show that the refractive index of the composite layer decreases with temperature. This trend suggests that the gas sheet of this composite layer has a dominant effect on the refractive index $\left(n_{3}\right)$ of the composite layer when the temperature increases. This may occur because the refractive index of gases generally decreases as the temperature increases. The decrease in the refractive index, however, is much less at atmospheric pressure than at higher pressures $(100,200$, and $400 \mathrm{psi})$. This effect of the pressure is associated with the divergence patterns in Figs. 2 and 3 where the oscillatory patterns exhibit little or no divergence at atmospheric pressure compared to the patterns at higher pressures.

\section{Refractive index for gases at high pressures and temperatures}

The refractive index of a gas, $n_{g}$, is related to its density by the following Gladstone-Dale (GD) relation: ${ }^{26}$

$$
n_{g}=1+R_{\mathrm{GD}} \rho,
$$

where $R_{\mathrm{GD}}$ is the Gladstone-Dale constant and $\rho$ is the density of the gas. The values of $R_{\mathrm{GD}}$ are 0.238 and $0.158 \mathrm{~cm}^{3} / \mathrm{g}$ for nitrogen and argon, respectively. Equation (7) can be rewritten for an ideal gas as follows:

$$
n_{g}=1+R_{\mathrm{GD}} \frac{P M}{R^{*} T},
$$

where $M$ is the molecular weight of the gas, $R^{*}$ is the universal gas constant, and $T$ is the absolute temperature of the gas. The theoretical refractive indices of the two gases obtained from Eq. (7) are plotted in Fig. 9, which also contains the refractive indices determined from the experimental data. The refractive index for the gases is obtained by considering that the refractive index of the composite layer depends on the refractive indices of $\mathrm{SiC}$ and gas linearly as given by

$$
n_{3}=a \times n+b \times n_{g},
$$

where $a$ and $b$ are two constants which are determined by fitting the data, i.e., the values of $n_{3}$ and $n$ (which are based on the experimental data) and the values of $n_{g}$ [which are based on the theoretical expression (8)], at two different temperatures such as the room temperature and $300{ }^{\circ} \mathrm{C}$. These values are listed in Table I for nitrogen and argon, respectively, for different pressures. The refractive index of the gas was found to be inversely proportional to temperature and directly proportional to the pressure of the gas, as predicted by the theory. The refractive indices of nitrogen and argon

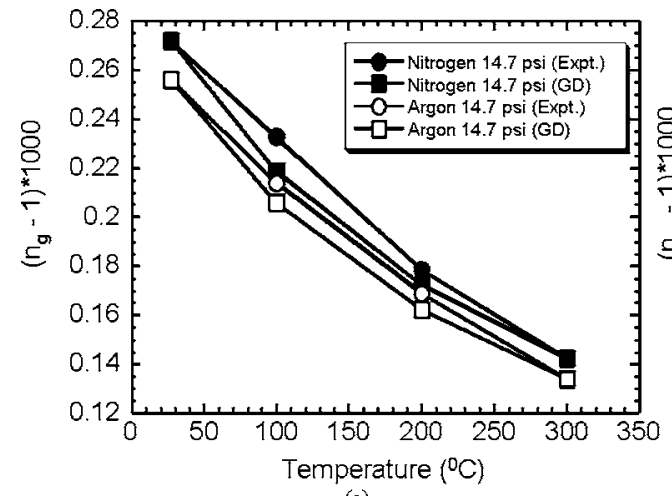

(a)

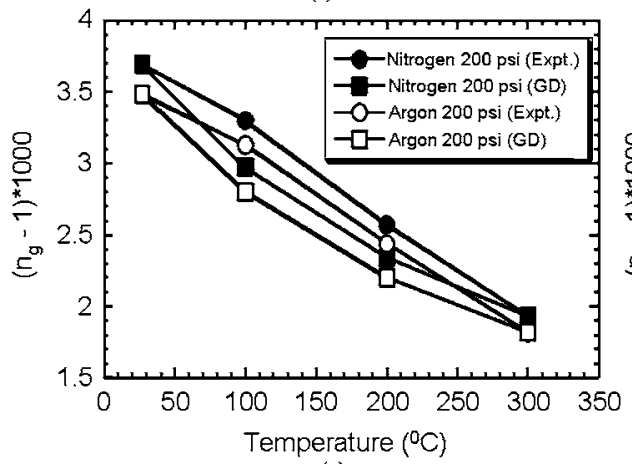

(c)

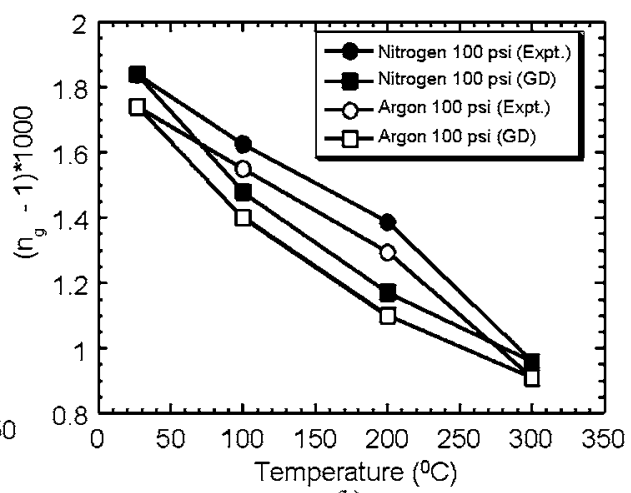

(b)

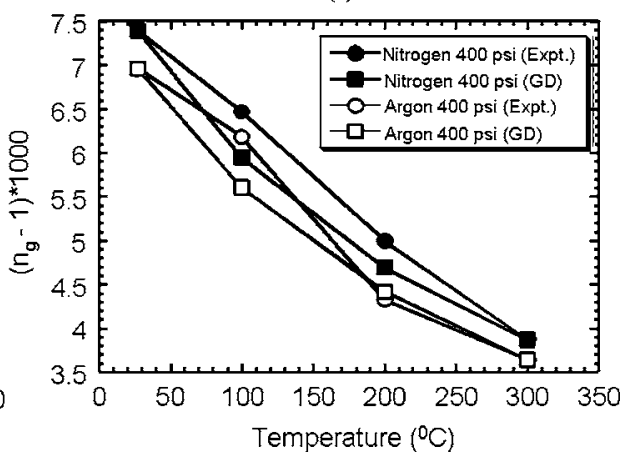

(d)
FIG. 9. Refractive indices of nitrogen and argon as a function of temperature from 27 up to $300{ }^{\circ} \mathrm{C}$ at pressures of (a) 14.7 , (b) 100 , (c) 200, and (d) 400 psi. 
TABLE I. Fitting parameters $a$ and $b$ for determining the refractive index of nitrogen and argon.

\begin{tabular}{|c|c|c|c|c|c|c|c|c|c|}
\hline \multirow[b]{2}{*}{ Gas } & \multirow{2}{*}{$\begin{array}{l}\text { Pressure } \\
\text { (psi) }\end{array}$} & \multicolumn{3}{|c|}{$27^{\circ} \mathrm{C}$} & \multicolumn{3}{|c|}{$300^{\circ} \mathrm{C}$} & \multirow[b]{2}{*}{$a$} & \multirow[b]{2}{*}{$b$} \\
\hline & & $n_{3}$ & $n$ & $n_{g}$ & $n_{3}$ & $n$ & $n_{g}$ & & \\
\hline \multirow[t]{4}{*}{ Nitrogen } & 14.7 & 2.359 & 2.41618 & 1.00027 & 2.34623 & 2.43851 & 1.00014 & -0.5411 & 3.665 \\
\hline & 100 & 2.35 & 2.42312 & 1.00184 & 1.99335 & 2.44037 & 1.00096 & -18.29 & 46.602 \\
\hline & 200 & 2.3284 & 2.42593 & 1.00369 & 1.98377 & 2.44312 & 1.00193 & -15.88 & 40.703 \\
\hline & 400 & 2.29255 & 2.42885 & 1.00789 & 1.9718 & 2.44729 & 1.00387 & -11.61 & 30.278 \\
\hline \multirow[t]{4}{*}{ Argon } & 14.7 & 2.2104 & 2.41202 & 1.00025 & 2.19345 & 2.43556 & 1.00013 & -0.6876 & 3.8676 \\
\hline & 100 & 2.20871 & 2.42020 & 1.00174 & 2.12064 & 2.43833 & 1.00091 & -4.283 & 12.552 \\
\hline & 200 & 2.19357 & 2.42206 & 1.00348 & 2.06 & 2.44020 & 1.00182 & -5.872 & 16.360 \\
\hline & 400 & 2.17981 & 2.4251 & 1.00696 & 1.98027 & 2.44385 & 1.00364 & -7.191 & 19.485 \\
\hline
\end{tabular}

are shown in Fig. 10 as a function of pressure. The gases do not show a significant variation in their respective refractive index at atmospheric pressure even when the temperature is increased. As the pressure increases, however, the temperature change induces a significant variation in the refractive indices for both gases.

\section{CONCLUSION}

The effect of gas on the optical properties of $6 \mathrm{H}-\mathrm{SiC}$ has been studied as a function of temperature and pressure. The reflectance of the $\mathrm{SiC}$ wafer exhibited unique oscillatory patterns for both nitrogen and argon at high pressures and tem- peratures. The oscillatory pattern tends to diverge with an increase in pressure. This divergence was more prominent for argon than for nitrogen. These patterns can be utilized to determine the refractive indices of $\mathrm{SiC}$ and the underlying gas in order to measure the pressure of the gas and to identify the type of the gas. At high pressures and temperatures, complex optothermal and optomechanical interactions occur between the gas and the compressed atomic planes of the $\mathrm{SiC}$ crystal, leading to the formation of a composite layer whose refractive index is different from that of the original $\mathrm{SiC}$ wafer segment and the underlying gas. The refractive index of this composite layer was obtained using the reflectance

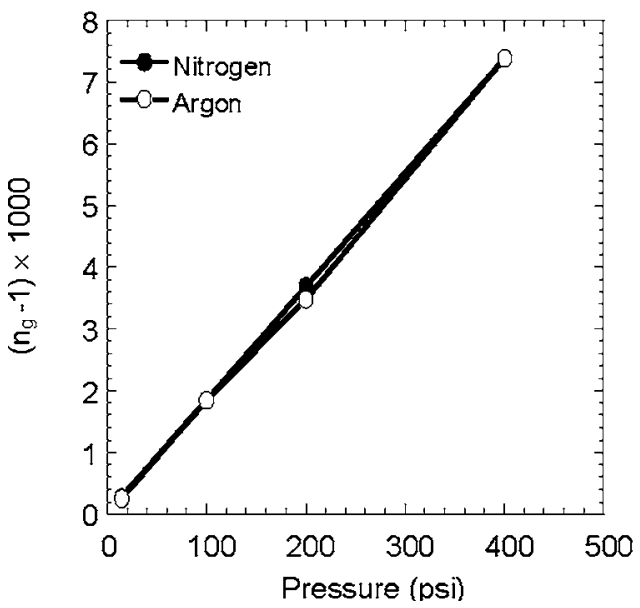

(a)

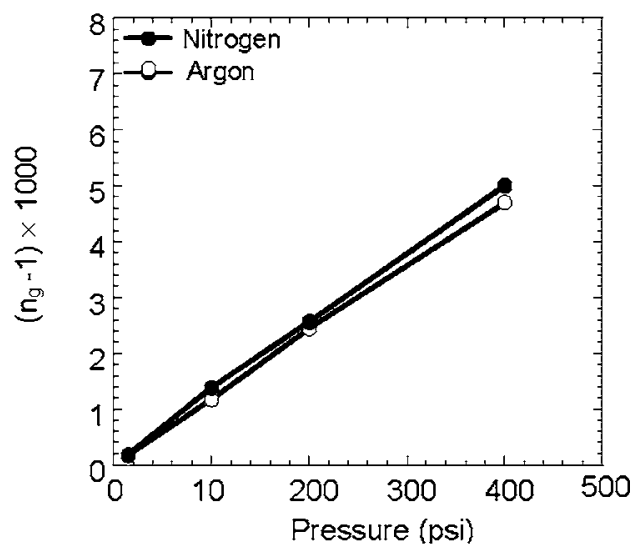

(c)

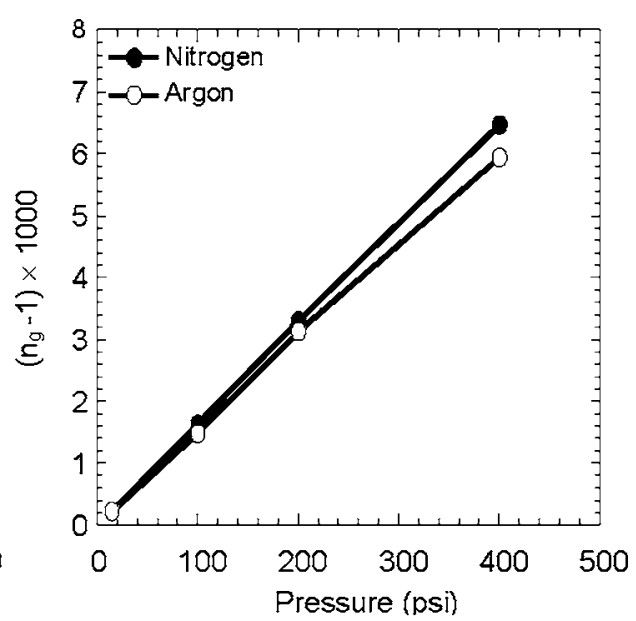

(b)

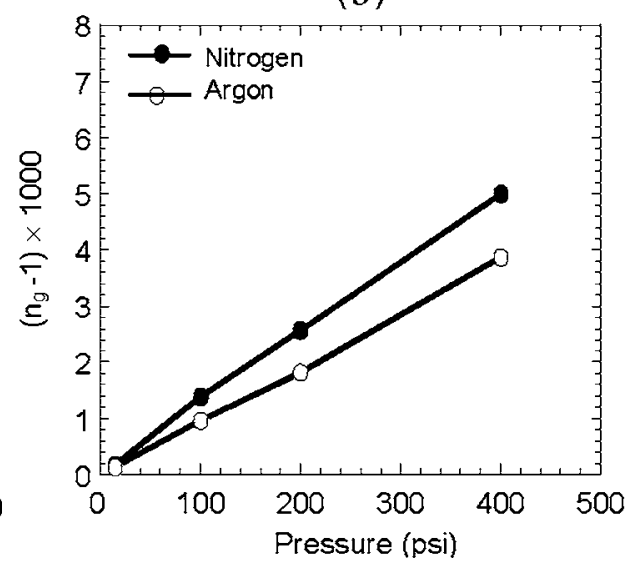

(d)
FIG. 10. Refractive indices of nitrogen and argon as a function of pressure for different temperatures: (a) 27, (b) 100 , (c) 200 , and (d) $300{ }^{\circ} \mathrm{C}$. 
data and then the refractive indices of the gases were obtained using an empirical relation involving the refractive indices of the composite layer and the $\mathrm{SiC}$ wafer segment.

\section{ACKNOWLEDGMENTS}

This work was supported by Nuonics, Inc., and the Florida Photonics Center of Excellence at the College of Optics and Photonics in the University of Central Florida. The overall project was sponsored by Susan Maley, Project Manager, U.S. Department of Energy, National Energy Technology Laboratory, Morgantown, West Virginia, and the authors appreciate her continued support for the project.

${ }^{1}$ L. Uneus et al., IEEE Sens. J. 5, 75 (2005).

${ }^{2}$ B. S. Kang, F. Ren, B. P. Gila, C. R. Abernathy, and S. J. Pearton, Appl. Phys. Lett. 84, 1123 (2004).

${ }^{3}$ C. K. Kim, J. H. Lee, Y. H. Lee, N. I. Cho, and D. J. Kim, Sens. Actuators B 66, 116 (2000).

${ }^{4}$ O. Bohme, A. L. Spetz, I. Lundstrom, and D. Schmeisser, Adv. Mater (Weinheim, Ger.) 13, 597 (2001).

${ }^{5}$ T. J. Fawcett, J. T. Wolan, R. L. Myers, J. Walker, and S. E. Saddow, Appl. Phys. Lett. 85, 416 (2004).

${ }^{6}$ P. Tobias, B. Golding, and R. N. Ghosh, IEEE Sens. J. 3, 543 (2003).

${ }^{7}$ G. Muller and G. Krotz, Sens. Actuators, A 43, 259 (1994).

${ }^{8}$ J. H. Visser and R. E. Soltis, IEEE Trans. Instrum. Meas. 50, 1543 (2001).

${ }^{9}$ M. E. Levinshtein, S. I. Rumyantsev, and M. S. Shur, Properties of Advanced Semiconductor Materials GaN, AlN, InN, BN, SiC, SiGe (Wiley, New York, 2001), pp. 93-147.
${ }^{10}$ J. Du, D. J. Young, C. A. Zorman, and W. H. Ko, Tech. Dig. - Int. Electron Devices Meet. 2003, 32.6.1.

${ }^{11}$ A. Arbab, A. Spetz, and I. Lundtrom, Sens. Actuators B 19, 562 (1994).

${ }^{12}$ H. Wingbrant, H. Svenningstrop, P. Salomonsson, D. Kubinski, J. H. Visser, and A. L. Spetz, Sensors, 2003. Proc. IEEE 1, 428 (2003).

${ }^{13} \mathrm{H}$. Wingbrant, H. Svenningstorp, P. Salomonsson, P. Tengström, I. Lundström, and A. L. Spetz, Sens. Actuators B 93, 295 (2003).

${ }^{14}$ A. Trinchi, K. Galatsis, W. Wlodarski, and Y. X. Li, IEEE Sens. J. 3, 548 (2003).

${ }^{15}$ K. Noda, M. Takahashi, R. Ohba, and S. Kakuma, Opt. Eng. 44, 014301 (2005).

${ }^{16}$ H. Tai, H. Tanaka, and T. Yoshino, Opt. Lett. 12, 437 (1987).

${ }^{17}$ P. I. Nikitin, A. A. Beloglazov, M. V. Valeiko, J. A. Creighton, and J. D. Wright, Rev. Sci. Instrum. 68, 2554 (1997).

${ }^{18}$ J. I. Nowicka, T. Pustelny, Z. Opilski, E. Maciak, W. P. Jakubik, and M. Urbanczyk, Opt. Eng. 40, 2978 (2003).

${ }^{19}$ J. A. P. Lima et al., Rev. Sci. Instrum. 71, 2928 (2000).

${ }^{20}$ L. Cheng, A. J. Steckl, and J. Scofield, IEEE Trans. Electron Devices 50, 2159 (2003).

${ }^{21}$ G. Cocorullo, F. G. Della Corte, and I. Rendina, Appl. Phys. Lett. 74, 3338 (1999).

${ }^{22}$ Z. Li and R. C. Bradt, in Silicon Carbide Symposium, edited by J. D. Cawley and C. E. Semler (American Ceramic Society, Columbus, OH, 1987), p. 313.

${ }^{23}$ W. F. F. Riley, L. D. Sturges, and D. H. Morris, Mechanics of Materials, 5th ed. (Wiley, New York, 1998).

${ }^{24}$ G. L. Harris, in Properties of Silicon Carbide, EMIS Data Reviews Series Vol. N13, edited by G. L. Harris (INSPEC, the Institution of Electrical Engineers, London, United Kingdom, 1995), p. 8.

${ }^{25}$ E. Hecht, Optics, 4th ed. (Pearson Education, Singapore, 2002).

${ }^{26}$ W. Merzkirch, Flow Visualization (Academic, New York, 1974), pp. 63-69. 